\title{
Comunicação organizacional à la française: avanços e perspectivas
}

\author{
À la française organizational communication: \\ advances and perspectives
}

Comunicación organizacional à la française: avances y perspectivas

\section{Alice Zozima Rego de Souza Paris}

- Doutoranda em Sciences de l'Information et de la Communication e pesquisadora vinculada ao Laboratoire d'Information, Milieux, Médias et Médiation (I3M), Université du Sud - Toulon - Var (USTV), em co-tutela com a Escola de Comunicações e Artes da Universidade de São Paulo (ECA-USP), sob a coorientação da Prof. Dra. Margarida M. Krohling K. Kunsch

- Master Recherche em (Dispositifs Socio-Techniques d'Information et de Communication (Distic) e em Sciences de l'Information et de la Communication na Université du Sud - Toulon - Var (França)

- Master II em Communication E-Redactionnel na Université du Sud - Toulon - Var

- Master I em Communication Internacional na Université de Nantes (França)

- Graduação em Jornalismo pela Universidade Católica de Pernambuco (Unicap)

- E-mail: alice_zozima@hotmail.com

\section{Larissa Conceição dos Santos}

- Doutora em Sciences de l'Information et de la Communication pela École des Hautes Études en Sciences de l'Information et de la Communication (Celsa) pelo Laboratoire Gripic, da Université de Paris-Sorbonne, em cotutela com a Escola de Comunicações e Artes (ECA-USP), sob a coorientação da Prof. Dra. Margarida M. Krohling Kunsch

- Mestre em Sciences de l'Information et de la Communication pelo Celsa, Université Paris-Sorbonne

- Mestre em Engenharia da Produção pela Universidade Federal de Santa Maria (UFSM)

- Bacharel em Administração e bacharel em Relações Públicas pela UFSM

- Professora substituta na Universidade Federal do Pampa (Unipampa)

- E-mail: larissa.conceicaos@gmail.com 


\section{Resumo}

0 fortalecimento do campo da comunicação organizacional em esfera mundial se deve, primeiramente, ao empenho, à dedicação e à pesquisa de investigadores implicados na formação de um arcabouço teórico e disciplinar originais. Em segundo lugar, salienta-se o diálogo e o intercâmbio necessários entre as perspectivas oriundas de diferentes partes do globo, em vista da legitimação do conhecimento científico. Nesse sentido, apresenta-se um panorama da comunicação organizacional na França, destacando a evolução da área, as principais linhas de investigação e os pesquisadores nelas implicados.

\section{PALAVRAS-CHAVE: ORGANIZAÇÕES • COMUNICAÇÃO ORGANIZACIONAL・RELAÇÕES PÚBLICAS・APROXIMAÇÕES TEÓRICAS・FRANÇA.}

\section{Abstract}

The strengthening of the field of organizational communication at the global level is due, firstly, to the commitment, to the dedication and to the research of investigators involved in the formation of a theoretical and disciplinary structure that is original. Secondly, one emphasizes the necessary dialogue and interchange between the perspectives coming from different parts of the world, in view of the legitimization of scientific knowledge. In this sense, one presents an overview of organizational communication in France, highlighting the evolution of the area, the main lines of research and the researchers involved with them.

\section{KEYWORDS: ORGANIZATIONS•ORGANIZATIONAL COMMUNICATION・PUBLIC RELATIONS・ THEORETICAL APPROACHES・FRANCE.}

\section{Resumen}

El fortalecimiento del campo de la comunicación organizacional en el ámbito mundial se debe, en primer lugar, al empeño, a la dedicación y a la investigación de estudiosos involucrados en la formación de un referencial teórico y disciplinario originales. En segundo lugar, se enfatiza el diálogo y el intercambio necesarios entre las perspectivas provenientes de diferentes partes del globo, en dirección a la legitimización del conocimiento científico. En ese sentido, se presenta un panorama de la comunicación organizacional en Francia, destacando la evolución del área, las principales líneas de investigación y los investigadores dedicados a ellas. 
ANO 13 • NÚMERO 24 • 1ํSEM. 2016 - ORGANICOM

COMUNICAÇÃO ORGANIZACIONAL À LA FRANÇAISE: AVANÇOS E PERSPECTIVAS

Et voilà que nous sommes en France, le pays du fromage et du vin!

$\mathrm{E}$ m termos de superfície, a França é quinze vezes menor que o Brasil, mas, em contrapartida, dentro de seus 675.000m (Carayol; Gramaccia, 2013) se escondem riquezas culturais incomparáveis. Entretanto, não estamos aqui para discutir sobre a cultura francesa nem sobre sua superfície. Este resumo foi só para dar um gostinho extra sobre o que iremos falar.

A diversidade francesa que aqui propomos abordar possui caráter intelectual e se refere à multiplicidade de panoramas e vieses pelos quais podemos observar a chamada "comunicação das organizações". 0 estudo de tal temática evoluiu ao longo das últimas décadas assumindo contornos teóricos e epistemológicos próprios ao contexto acadêmico francês, buscando diferenciar-se das tradições funcionalistas e tecnicistas da comunicação em direção à formação de abordagens interpretativas, críticas e, acima de tudo, interdisciplinares (Bouzon, 2011).

0 desenvolvimento do campo da comunicação organizacional no seio das ciências da informação e da comunicação na França, diferentemente de outros países onde ela se vê vinculada sobretudo à área de comunicação, torna seu escopo e sua atuação diferenciados, conforme salienta Bouzon (2011, p. 17): "la problemática tratada es múltiple (...). Se aborda tanto el contenido y el lugar que concierne a la información (y su tratamiento), como a las modalidades de los actos comunicativos o al papel de estos últimos en las situaciones de trabajo".

Ou seja, falar em comunicação organizacional na França torna-se um tema delicado, pois a própria definição da expressão - comunicação das organizações, para uns, e comunicação organizacional, para outros - suscita ainda grandes discussões. Encontraremos diferentes nomenclaturas e as mais variadas significações a elas atribuídas, sem um consenso, aparente, ou uma definição geral englobante.

De tal forma que, em recente publicação (Carayol; Gramaccia, 2013), dirigida pelos coordenadores da revista Communication \& Organisation, visando constituir uma genealogia das temáticas recorrentes e formar uma arqueologia do saber do campo da comunicação organizacional, não se apresenta uma definição precisa das expressões communication organisationnelle ou communication des organisations. Uma tal imprecisão poderia ser explicada pela diversidade de perspectivas e de pesquisadores nelas implicados, o que buscaremos analisar a seguir, a partir de uma breve cartografia dessa área na França.

A pesquisa francesa, em comunicação organizacional, foi capaz de superar a influência positivista proeminente na década de 1980, oriunda das correntes norte-americanas, ${ }^{2}$ e as correntes mecanicistas do final da década de $1950^{3}$, e, a partir do intercâmbio entre as investigações em ciências humanas e sociais ${ }^{4}$, promover abordagens singulares e originais para 0 estudo da relação complexa entre comunicação e organização.

No âmbito do presente estudo, busca-se, então, apresentar o cenário da comunicação organizacional francesa, suas origens, a evolução do campo e as principais linhas de investigação, bem como seus representantes, visando trazer à luz diferentes abordagens à comunidade acadêmica brasileira.

1 E aqui estamos nós na França, o país do queijo e do vinho!

2 Como, por exemplo, as abordagens desenvolvidas por W. Charles Redding.

3 Oriundas das ciências da gestão e da teoria da organização, como as perspectivas de James G. March e Herbert A. Simon.

4 Por exemplo: Foucault, Crozier, Bourdieu, Cornelius Castoriadis, Jean-François Lyotard, entre outros. 


\section{COMUNICAÇÃO ORGANIZACIONAL NA FRANÇA: ANTECEDENTES, PRECURSORES E ORIGENS}

Como toda história tem uma origem - e toda origem, uma história -, os estudiosos da comunicação organizacional, no início de suas pesquisas, e à procura de uma perspectiva de legitimação científica dentro dessa disciplina, levantam várias questões sobre a circulação das teorias e seus conceitos. Essas questões surgiram por sua grande influência interdisciplinar relacionada à gestão, à administração, às ciências humanas e sociais e, especialmente, às ciências da informação e da comunicação. Encontraremos essas mesmas influências no campo histórico brasileiro e norte-americano, principalmente no que se diz respeito à mudança de nomenclatura da chamada comunicação institucional, corporativa ou de empresa, em direção à formação da área que hoje denominamos comunicação organizacional.

$\mathrm{Na}$ França, tudo começa com o aparecimento da revista Humanisme et Entreprise ${ }^{5}$, no início dos anos 1960. Essa publicação teria uma grande influência nos discursos e nas técnicas dos empreendedores com relação à mudança organizacional. A revista contava com a participação de autores acadêmicos, que faziam parte do domínio da literatura e das ciências humanas, e de líderes empresariais, nacionais e internacionais.

Paralelamente à publicação dessa revista, dois outros eventos irão marcar o cenário francês: por um lado, a primeira aliança entre atores empresariais e acadêmicos, estruturada, sobretudo, por profissionais das áreas de relações sociais, relações públicas, publicidade e marketing; por outro, a criação de novos cursos universitários, embora os pesquisadores se façam discretos.

Veremos que, de 1960 até os anos 1990, diferentes teorias eáreas de pesquisas simbolizam a diversidade de sujeitos publicados por essa revista, como se pode constatar através do estudo realizado em 1998 pela professora Françoise Bernard ${ }^{6}$, ao analisar grande parte dos artigos da revista Humanisme et Entreprise. De acordo com Bernard (1998), entre 1960 e 1970, os temas publicados são relacionados a patologia, psicologia, cibernética, dinâmica de grupo e técnicas de relações humanas. As empresas confrontam-se, nesse período, com conflitos e insatisfações. Trata-se de uma fase de turbulência, assim qualificado por Bernard (1998). Os artigos publicados tratam também de economia, consumismo, empresa não-amada, o mal-estar entre os chefes, entre outros. Também são observadas temáticas relativas à mudança cultural entre os funcionários.

Entre 1970 e 1980, é a sociologia que ocupa um lugar de destque. Dessa vez, destaca-se a importância do homem no setor industrial. Esse período será marcado por protestos e críticas sociológicas, psicológicas e antropológico-econômicas. Isso quer dizer que a empresa é eminentemente um lugar de troca. A revista vai publicar artigos que dizem respeito à economia e felicidade, à ruptura entre o indivíduo e o trabalho.

Entre 1980 e 1990, dá-se início ao reconhecimento do ambiente à aceitação do conceito da organização comunicante, relacionada ao acesso à rede e à multiplicidade de relações (indivíduos, grupos, suportes, métodos, tecnologia etc.). Tais conceitos irão

5 A revista Humanisme et Entreprise (Humanismo e Empresa) foi criada em 1959 pelo professor Charles-Pierre Guillebeau, presidente da Associação dos Antigos Alunos de Letras e Ciências Humanas da Universidade de Paris (AAELP) e fundador da École des Hautes Études en Sciences de l'Information et de la Communication (Celsa), da Université de Paris-Sorbonne, durante uma palestra de abertura na Sorbonne, sobre o mundo econômico e social. Destinada inicialmente aos literários e desejando inserir-se no mundo corporativo, eles inauguraram a aproximação entre a universidade e a empresa, o que levou, alguns anos mais tarde, à criação da Celsa. Humanisme et Entreprise é uma revista acadêmica multidisciplinar referenciada nas instâncias acadêmicas francesas. Trata-se de uma das mais antigas revistas francófonas dedicadas à pesquisa sobre a vida corporativa e ao pensamento de seus líderes.

6 Françoise Bernard, professora de ciências da informação e da comunicação na Universidade de Aix-Marseille e dirigente do Instituto de Investigação em Ciências da Informação e da Comunicação (Irsic EA 4262). presidente honorária da Société Française des Sciences de l'Information et de la Communication (Sfisic), é atualmente a primeira vice-presidente da seção 71 do Conseil National des Universités (CNU). Especialista em comunicação das organizações e das instituições, trabalha e publica sobre temas relacionados a mudança organizacional, reconfigurações e transições em relação às formas organizacionais, sociais e culturais. 
ANO 13 • NÚMERO 24 • 1ํSEM. 2016 - ORGANICOM

COMUNICAÇÃO ORGANIZACIONAL À LA FRANÇAISE: AVANÇOS E PERSPECTIVAS

influenciar cada vez mais os estudos em ciências da informação e da comunicação, os quais, por sua vez, serão confrontados por outras duas disciplinas: as novas tecnologias de informação de comunicação (NTIC) e as redes (Bernard, 1998a, p. 6).

A revisão histórica e interdisciplinar que acabamos de tecer torna-se fundamental para a compreensão das mudanças no campo da comunicação institucional, e o seu fortalecimento enquanto prática e também como disciplina. Nessa perspectiva, a comunicação corporativa tenta criar condições para a aceitação de mensagens e ações planejadas pela administração da empresa.

De acordo com o professor Christian Le Moënne ${ }^{7}$, esse período também foi marcado pelo desenvolvimento massivo de "agências de comunicação", a grande maioria derivada

da evolução das agências de relações públicas ou agências de publicidade. Finalmente, nota-se a criação e o desenvolvimento, a partir de 1985, de muitas associações profissionais de comunicação, o que irá contribuir para a construção da legitimidade dos deveres profissionais dos "comunicadores" (Le Moënne; Gallot, 2015, p. 5).

É a partir de 1992 que as áreas de comunicação institucional e corporativa, muitas vezes limitados às práticas gerenciais, institucionais e comerciais, serão redefinidos, deixando, enfim, espaço para a comunicação organizacional, que emerge como prática, mas também como disciplina acadêmica. Na prática, ela se concentra nas atividades tradicionais da empresa, ou seja, na gestão de recursos humanos, na atividade comercial e nas relações públicas. Já no campo teórico, ela vai finalmente ter um lugar com o surgimento da revista acadêmica Communication \& Organisation, criada pela Sociedade Francesa de Ciências da Informação e da Comunicação (Sfsic). Essa publicação, de acordo com Bernard (1998), causa uma ruptura com a revista anterior, a Humanisme et Entreprise, dando continuidade à legitimação dos atores profissionais de comunicação, mas também acadêmicos.

Com o lançamento de uma nova publicação, destinada exclusivamente aos pesquisadores consagrados aos estudos em língua francesa sobre a comunicação organizacional, e à sua consequente visibilidade, a comunicação organizacional na França começa lentamente a se desenvolver como um campo científico, respaldado diretamente pelos membros da Sfsic.

\section{LEGITIMAÇÃO E INSTITUCIONALIZAÇÃO DO CAMPO}

De acordo com Nicole D'Almeida ${ }^{8}$ e Yanita Andonova9 (2008), a legitimidade do campo prático e profissional da comunicação nas e das organizações francesas não foi acompanhada pela legitimação do campo científico, como acabamos de ver. Por um lado, observa-se a propagação de atividades comunicacionais no interior das organizações e o crescente interesse de investigadores, de diferentes campos do saber, que se servem de tais processos como objetos de estudo. Por outro, evidencia-se 0 contraste entre as análises puramente descritivas e finalidades funcionais, e a validade científica para a construção de um campo de pesquisa sólido, pois

7 Christian Le Moënne é professor de Ciências da Informação e da Comunicação da Universidade de Rennes 2 e pesquisador Cersic-Erellif, presidente honorário da Sfisic, criador do Grupo de Estudo e Pesquisa em Comunicação Organizacional - "Org \& Co" e cofundador, com Gino Gramaccia, da Revue Française des Sciences de l'Information et de la Communication - www.rfsic.org.

8 Nicole D'Almeida é professora de Ciências da Informação e da Comunicação no Celsa (Universidade de Paris-Sorbonne), onde ensina e dirige a formação e a pesquisa nas seguintes áreas: comunicação interna e externa, histórias organizacionais, dinâmica de opinião, comunicação de meio ambiente, desenvolvimento sustentável e responsabilidade social empresarial.

9 Yanita Andonova é professora e vice-diretora da Faculdade de Ciências da Comunicação na Universidade Paris 13 e pesquisadora do LabSic. 
ANO 13 • NÚMERO 24 • 1픈. 2016 • ORGANICOM

COMUNICAÇÃO ORGANIZACIONAL À LA FRANÇAISE: AVANÇOS E PERSPECTIVAS

é nesse contexto, marcado pelo duplo circuito das contribuições à eficácia das empresas e da investigação sobre objetos considerados legítimos no plano científico, que se faz necessário rever a atual abordagem da comunicação organizacional" (D'Almeida; Andonova, 2008, p. 34).

E, se bem que o début das atividades de comunicação organizacional na França remeta, entre outros fatores, ao surgimento da primeira diretoria de Comunicação (empresarial) na Saint-Gobain, em 1971, no campo científico podemos ressaltar 0 ano 1994 como marco da criação do primeiro grupo de pesquisas nacional reunindo investigadores franceses dedicados à temática da comunicação organizacional, denominado Org \& Co ${ }^{10}$. Esse grupo tem como objetivo principal ajudar a estruturar, no país, um campo de pesquisa em comunicação organizacional, confrontando seus problemas, metodologias, olhares, abordagens e resultados mediante a troca de conhecimentos e métodos de investigação. Nesse ponto, pode-se afirmar que "o estudo da comunicação das organizações se estendeu, assim, da observação dos fenômenos comunicacionais no seio das organizações, e à análise das organizações pela comunicação"11 (Bouillon et al., 2007, p. 7).

Segundo Fabienne Martin-Juchat (2012, p. 184)12, os principais questionamentos desse grupo, inicialmente, resumiam-se a:

1. Qual é literatura sobre organização com relação à comunicação?

2. Quais os modelos de comunicação praticados pelas organizações?

3. Quais os modelos sobre a relação entre informação, comunicação e organização?

A partir de2002, apósuma pergunta realizada por Françoise Bernard sobre a relação ontológica entre aorganização e comunicação, os investigadores concluíram que seria necessário aumentar o questionário:

4. A comunicação cumpre um papel de institucionalização, de estruturação das organizações?

5. A comunicação constrói a realidade organizacional, o que é possível observar através dela?

0 processo de legitimação do campo de pesquisa da comunicação organizacional, no ambiente acadêmico francês, tem incío com a publicação, em 2002, do artigo de Françoise Bernard intitulado "Contribuição para uma história da comunicação das organizações dentro das ciências da informação e da comunicação" (Bernard, 2002). Mesmo assim, essa disciplina ainda é considerada como uma recém-chegada, isto é, a comunicação organizacional na França encontra-se ainda em uma fase de consolidação histórica, diferente do que podemos ver nos Estados Unidos, no Canadá, e mesmo no Brasil.

Na França, a denominação "comunicação organizacional" serve, por enquanto, para reconhecer ou fazer referência a um domínio comum. Alguns pesquisadores defendem esse impasse, afirmando que sua história ainda está sendo escrita e, por causa disso, não está muito bem traçada. Le Moënne salienta que "a ciência está a sendo feita" (Le Moënne; Gallot, 2015, p. 3). 0 autor afirma que os investigadores franceses ainda estão muito reticentes para escrever sua própria história, porque há muitas interrogações teóricas e institucionais dentro da pesquisa em comunicação organizacional francesa. 0 estudioso não esconde a necessidade de se desenvolver uma cronologia histórica para os jovens investigadores. Entretanto, ele assume que essa tarefa é um pouco complicada. Para ele, "não é costume pedir aos historiadores escreverem sua própria história. Vemos nesse caso um ligeiro viés metodológico. Tentar uma revisão crítica da pesquisa em comunicação organizacional na França é uma operação intelectualmente delicada" ( Le Moënne; Gallot, 2015, p. 3).

Françoise Bernard, por sua vez, está de acordo com a possibilidade de um estudo mais aprofundado da história da comunicação organizacional. Para ela, esse estudo

100 grupo comporta hoje quase duzentos pesquisadores de diferentes países francófonos (França, Bélgica, Canada, Suíça, Tunísia, entre outros).

11 Tradução livre nossa.

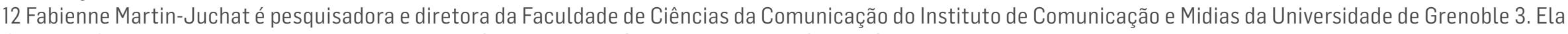
é responsável pelo Programa de Pesquisa sobre as Comunicações Organizacionais no Centro Gresec. 
poderia atualizar os modos de tratamento da gestão simbólica tal como eles foram projetados e instrumentados, como, por exemplo, depois da invenção da indústria. Tal projeto incluiria a gestão simbólica gerencial praticada desde o início dos anos 1980 como uma forma de gestão do simbolismo histórico, numa perspectiva de continuidade e ruptura com os especialistas em comunicação organizacional em relação aos estudos históricos: monografias, história do marketing, história da contabilidade, história e cultura corporativa etc. (Bernard, 1998, p. 52).

Em outras palavras, os professores Le Möenne e Bernard fazem parte de um grupo de pesquisadores que pensam que um balanço histórico sobre a pesquisa da comunicação organizacional é imprescindível e necessário. 0 mesmo declaram outros pesquisadores, como Nicole D'Almeida, Gino Gramaccia ${ }^{13}$ e Pierre Delcambre ${ }^{14}$, que por esse motivo organizaram um colóquio ${ }^{15}$ na cidade de Rennes, para marcar o 30 aniversário da criação do grupo Org \& Co e discutir sobre os "trinta anos de pesquisa sobre comunicação organizacional na França". As questões expostas como ancoragem foram: qual o balanço de investigações sobre as práticas de informação e comunicação organizacional? Quais as questões e orientações teóricas? Quais as comparações internacionais? Quais as pesquisas emergentes?

\section{DE NORTE A SUL: UMA CARTOGRAFIA DA COMUNICAÇÃO ORGANIZACIONAL}

A variedade de perspectivas reflete também a multiplicidade de enfoques teóricos e correntes (oriundas de diferentes disciplinas) aos quais estão associados os principais pesquisadores da área, calcando-se, segundo Bouzon (2011), em um pluralismo explicativo que marca o campo da comunicação organizacional francesa. Na visão de D'Almeida e Andonova (2008, p. 41), "o campo da pesquisa em comunicação organizacional não é marcado pela unidade, mas antes pela convergência. $A$ unidade do objeto é apenas aparente e conduz a realidades e propósitos muito distintos".

Assim, coma consolidação das pesquisas em comunicação organizacional ampliou-se o número de pesquisadores em informação e comunicação dedicados a essa temática, formando uma comunidade acadêmica ampla e heterogênea, representativa em todas as regiões francesas. Entre os principais expoentes desse campo, no cenário francês, destaca-se aqui especialmente a atuação de Christian Le Moënne (Rennes), Françoise Bernard (Aix-Marseille), Gino Gramaccia (Bordeaux), Alex Muchielli (Montpellier), Pierre Delcambre (Lille), Arlette Bouzon (Toulouse), Jacques Bonnet (Grenoble) e Nicole d'Almeida (Paris).

Christian Le Moënne é professor de ciências da informação e da comunicação na Université de Rennes 2 e pesquisador no Cersic-Erellif. Suas investigações abordam a formação das instituições sociais e a institucionalização das empresas, além da maneira como elas estruturam as relações profissionais sobre o modelo da vida privada, valores, tradições partilhadas, normas organizacionais e mudanças.

Françoise Bernard, é professora de ciências da informação e da comunicação na Université Aix-Marseille e no Laboratoire Irsic desenvolve pesquisas sobre a comunicação organizacional em interface com as temáticas ambientais e a chamada

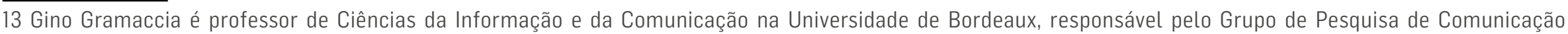

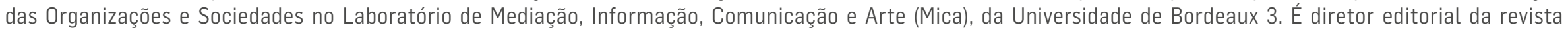
Communication \& Organisation e presidente honorário da Sfisic.

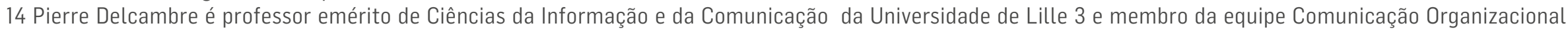

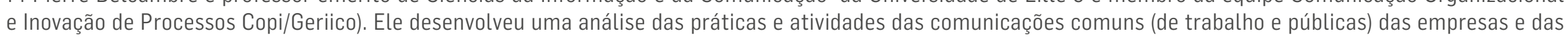
organizações (trabalho social, estaleiros, marinha mercante, do setor cultural e do mundo do espetáculo).

15 Colóquio "Trente ans de recherches sur les communications organisationnelles en France", realizado na cidade de Rennes, nos dias 10,11 e 12 de março de 2016. 
communication engageante, inspirada na psicologia social. Suaabordagemapoia-seespecialmentena pesquisa-ação, refutando o funcionalismo e as vertentes tecnicistas da comunicação e em prol da formação de uma "inteligência comunicacional".

0 pesquisador Gino Gramaccia (2015), professor de ciências da informação e da comunicação na Université Bordeaux 3 e responsável pelo Grupo de Pesquisa em Comunicação das Organizações e Sociedades junto ao Laboratório de Mediação, Informação, Comunicação e Arte (Mica), investiga a comunicação organizacional a partir da perspectiva dos atos de linguagem ${ }^{16}$, analisando os desafios e problemas organizacionais que limitam a ação humana, como a autonomia. Observa especialmente a comunicação desenvolvida na esfera operacional (ligada às tarefas) da atividade empresarial.

Inspirado nas correntes construtivistas e na teoria dos sistemas ${ }^{17}$. Alex Mucchielli (1998), diretor-fundador do Centre d'Étude et de Recherche en Information et Communication (Ceric) da Université Paul-Valéry de Montpellier III, apresenta uma perspectiva sistêmica e comunicacional das organizações. 0 autor observa a comunicação organizacional a partir dos rituais que permeiam as interações no interior das empresas. A comunicação diz respeito a todas as formas de expressão perceptíveis pelos atores organizacionais e passíveis de apropriação e ressignificação, por meio de jogos relacionais articulados que dão lugar à criação de sentido e garantem o funcionamento das organizações.

As relações entre comunicação e trabalho são o foco de investigação em comunicação organizacional conduzidas por Pierre Delcambre (2000), professor emérito de ciências da Informação e da Comunicação na Universidade de Lille 3 e membro da equipe Communications Organisationnelles et Processus d'Innovation (Copi), do Laboratório Groupe d'Études et de Recherche Interdisciplinaire en Information et Communication (Geriico). Suas pesquisas privilegiam a abordagem da sociologia do trabalho e da comunicação nas relações e interações no interior das organizações, bem como o lugar da linguagem na mediação das trocas interpessoais.

Arlette Bouzon (2006), pesquisadora no Laboratoire d'Études et de Recherches Appliquées en Sciences Sociales (Lerass), na Universidade de Toulouse, aborda a comunicação organizacional focalizando especialmente a inovação empresarial (inovação industrial e concepção colaborativa de produtos), a decisão e a inteligência coletiva, assim como dedica-se a estudos voltados à epistemologia e à construção teórica do campo acadêmico da comunicação organizacional.

Da região da Bourgogne, destacam-se os trabalhos de Jacques Bonnet (2015), professor de ciências da informação e da comunicação e diretor da equipe de pesquisa Communication, Savoirs, Médiations, Organisations (Cosmos), ligada ao Laboratoire en Sciences de l'Information et de la Communication (Cimes), da Universidade de Bourgogne. Suas pesquisas em comunicação organizacional têm como centro de interesse a construção partilhada de sentidos e saberes nas organizações, alcançados pela mediação do conhecimento e do diálogo coletivo, em prol da formação do que ele denomina "inteligência do social".

O estudo da comunicação interna, bem como as representações e interações comunicacionais, simbólicas e discursivas, são objeto de estudo da professora e pesquisadora Nicole d'Almeida da École des Hautes Études em Sciences de l'Information et de la Communication (Celsa) da Universidade de Paris-Sorbonne. Destaca-se, sobretudo, a perspectiva narratológica da comunicação organizacional proposta pela autora, que traz um viés inovador ao observar as organizações como espaços de construção e circulação de narrativas e, também, analisar as formas de "recitação" (narração) organizacional pelas quais as organizações se comunicam com os diversos públicos. 


\section{CONSIDERAÇÕES FINAIS}

Nos últimos dez anos a área de comunicação organizacional na França triplicou, aumentando de 30 mil para mais de 100 mil profissionais $^{18}$, demonstrando um volume crescente de negócios.

Nicole d'Almeida assevera que, "no âmbito acadêmico, a diversidade é a regra", pois se podem encontrar associações internacionais de pesquisadores que consideram os campos de relações públicas e de comunicação organizacional como dois setores distintos, como é o caso da International Communication Association (ICA). Outras cujo grupo de trabalho tem a designação "comunicação organizacional e estratégica", como a European Communication Research and Education Association (Ecrea) ou uma união dos setores da "comunicação organizacional e das relações públicas", como a Asociaciín Lationoamericana de Investigadores de la Comunicación (Alaic). Já na European Public Relations Education and Research Association (Euprera), embora o nome da associação se refira a relações públicas, os pesquisadores afirmam que fazem parte tanto destas quanto da comunicação organizacional (d'Almeida, 2015, p. 1).

Outro ponto fundamental refere-se à necessidade de fortalecimento da comunidade acadêmica e das redes de cooperação internacionais em comunicação organizacional. Essa troca de experiência mostra que, mesmo nos países com know-how em comunicação organizacional - como é o caso dos Estados Unidos e do Canadá e os "emergentes" França e Brasil -, a temática da comunicação nas organizações ainda apresenta grandes desafios. Ao comparar a comunicação no Brasil e na França D'Almeida esclarece que, "ao contrário da tradição francesa do tipo internalista, a abordagem neste caso é global ou integrada, todos focados nos aspectos macro e micro organizacionais, o importante é pensar em um sistema de relações, interferência e expressão" (d'Almeida, 2015, p. 3).

Nesse sentido, as perspectivas brasileiras não se distanciam das observadas no cenário gaulês. Alguns pesquisadores franceses referenciados no presente artigo já participaram de eventos no Brasil, como é o caso de Arlette Bouzon, Gino Gramaccia, Jacques Bonnet e Nicole d'Almeida. Esses intercâmbios entre pesquisadores "de part et d'autre de l'Atlantique"19, como se refere d'Almeida (2015) aos sul-americanos, ou "de outros mares" se fizermos nossa própria analogia, são essenciais para que continuemos a ver e a desenvolver uma pesquisa rica em experiências compartilhadas por outros países.

Finalmente, na esteira das pesquisas de Heller et al. (2013), defende-se a necessária evolução de uma postura crítica na abordagem da comunicação nas organizações rumo à adoção de uma perspectiva crítica, como condição de existência de pesquisas que contribuam para a consolidação do campo da comunicação organizacional e a reflexão acerca de seus objetos, dinâmicas e atores (Broise; Chantraine, 2013).

Por este trabalho, buscou-se apresentar, breve e sucintamente, um panorama da Comunicação Organizacional na França, esperando, com isso, contribuir com a discussão acadêmica internacional e fomentar o diálogo entre as perspectivas francesas e brasileiras, tão próximas e complementares, mas, por vezes, desconhecidas ou pouco exploradas, tendo em vista as barreiras linguísticas e editoriais que dificultam sua circulação.

\section{REFERÊNCIAS}

BERNARD, Françoise. La communication organisationnelle parcours vers une légitimitéscientifique. Tome 4. HDR, Université de Grenoble III, 1998. 
ANO 13 • NÚMERO 24 • 1은. 2016 - ORGANICOM

COMUNICAÇÃO ORGANIZACIONAL À LA FRANÇAISE: AVANÇOS E PERSPECTIVAS

\section{Grenoble III, 1998a.}

. Contribution à une histoire de la communication des organisations dans les SIC. In: BOURE, Robert. Les origines des sciences de l'information et de la communication, regards croisés. Villeneuve d'Asq : Presses Universitaires du Septentrion, 2002.

La communication des organisations entre questions d'influence et questions d'autonomie. L'actualité des notions d'engagement, d'émergence et d'institution. Communication \& Organisation, Presses Universitaires de Bordeaux, n. 47, p. 85-95, 2015.

BONNET, Jacques. L'intelligence du social dans les organisations. Communication \& Organisation, Presses Universitaires de Bordeaux, n. 47, p. 63-74, 2015.

BOUILLON, Jean-Luc, BOURDIN, Sylvie; LONEUX, Catherine. De la communication organisationnelle aux "approches communicationnelles" des organisations: glissement paradigmatique et migrations conceptuelles. Communication \& Organisation, Presses Universitaires de Bordeaux, n. 31, p. 07-25, 2007.

BOUZON, Arlette. Las investigaciones en comunicación de las organizaciones: orígenes y fundamentos. Organicom-Revista Brasileira de Comunicação Organizacional e Relações Públicas, a. 8, n.14, p. 12-28, 2011.

BROISE (de la), Patrice; CHANTRAINE, Olivier. De la position critique comme condition d'existence d'une recherche en communication organisationnelle. In: HELLER, Thomas et al. (ed). Communication \& Organisation: perspectives critiques. Villeneuve d'Asq: Presses du Septentrion, p. 389-400, 2013.

CARAYOL, Valérie. Communication organisationnelle, une perspective allagmatique. Paris: L'Harmattan, 2004.

CARAYOL, Valérie; GRAMACCIA, Gino. Recherches émergentes en communication des organisations. Communication \& Organisation, n. 47, p. 5-9, 2015.

(dir.). Abecedaire: vingt ans de recherches et de publications en communication des organisations. Bordeaux: PUB, 2013.

D'ALMEIDA, Nicole. La communication organisationnelle, de part et d'autre de l'Atlantique. Revue Française des Sciences de l'Information et de la Communication, n. 7, 2015. D0l:10.4000/rfsic.1574.

Les promesses de la communication. Paris: Presses Universitaires de France, 2001.

D'ALMEIDA, Nicole; ANDONOVA, Y. A comunicação das organizações. Anuário Unesco/Metodista de Comunicação Regional, a. 12, n. 12, p. 31-42, jan./dez. 2008.

DELCAMBRE, Pierre (dir.). Communications organisationnelles: objets, pratiques, dispositifs. Rennes: Presses Universitaires de Rennes, 2000.

GRAMACCIA, Gino. Les actes de langage dans les organisations. Paris: Editions L'Harmattan, 2001.

HELLER, Thomas et al. (eds.). Communication \& organisation: perspectives critiques. Villeneuve d'Asq: Presses du Septentrion, 2013. 
LE MOËNNE, Christian; GALLOT, Sidonie. Les recherches en communication organisationnelle en France: quelques éléments de bilans et de perspectives. Communiquer-Revue de Communication Sociale et Publique, n. 13, p. 123-43, 2015. DOI:10.4000/communiquer.1586.

MARTIN-JUCHAT, Fabienne. Acquis scientifique sur la communication organisationnelle: Repérage de points de convergence entre disciplines. In: MIEGE, Bernard; VINCK, Dominique, Les masques de la convergence: enquêtes sur sciences, industries et aménagements. Paris: Editions des archives contemporaines, 2012. p. 181-195.

MUCCHIELLI, Alex. Approche, systémique et communicationnelle des organisations. Paris: Armand Colin, 1998.

Artigo recebido em 01.03.2016 e aprovado em 06.05.2016. 